Detecting white dwarf binaries in Mock LISA Data Challenge 3

This article has been downloaded from IOPscience. Please scroll down to see the full text article.

2009 Class. Quantum Grav. 26204023

(http://iopscience.iop.org/0264-9381/26/20/204023)

View the table of contents for this issue, or go to the journal homepage for more

Download details:

IP Address: 194.94.224.254

The article was downloaded on 11/06/2010 at 10:23

Please note that terms and conditions apply. 


\title{
Detecting white dwarf binaries in Mock LISA Data Challenge 3
}

\author{
A Błaut ${ }^{1}$, A Królak ${ }^{2,3}$ and S Babak ${ }^{4}$ \\ ${ }^{1}$ Institute of Theoretical Physics, University of Wrocław, Wrocław, Poland \\ ${ }^{2}$ Institute of Mathematics, Polish Academy of Sciences, Warszawa, Poland \\ ${ }^{3}$ The Andrzej Soltan Institute for Nuclear Studies, Świerk-Otwock, Poland \\ ${ }^{4}$ Albert Einstein Institute, Golm, Germany \\ E-mail: ablaut@ift.uni.wroc.pl
}

Received 11 May 2009, in final form 21 July 2009

Published 6 October 2009

Online at stacks.iop.org/CQG/26/204023

\begin{abstract}
We present a strategy for detecting gravitational wave signals from the Galactic white dwarf binaries in the Mock LISA Data Challenge 3 (MLDC3) and estimate their parameters. Our method is based on the matched filtering in the form of the $\mathcal{F}$-statistic. We perform the search on three-dimensional space (sky coordinate and frequency of gravitational wave) below $3 \mathrm{mHz}$ and include the fourth parameter (frequency derivative) at high frequencies. A template bank is used to search for the strongest signal in the data, then we remove it and repeat the search until we do not have signals in the data above a preselected threshold. For the template bank, we construct an optimal grid that realizes the best lattice covering with a constraint such that the nodes of the grid coincide with the Fourier frequencies. This enables the use of the fast Fourier transform algorithm to calculate the $\mathcal{F}$-statistic.
\end{abstract}

PACS numbers: $04.80 . \mathrm{Nn}, 95.55 . \mathrm{Ym}$

(Some figures in this article are in colour only in the electronic version)

\section{Introduction}

The Galaxy contains a very large number of close binaries consisting of white dwarfs and/or naked helium stars. Compact binary sources populate the whole frequency band of the LISA detector. The most common sources are white-dwarf/white-dwarf (WDWD) binaries emitting gravitational wave signals of nearly constant frequency and amplitude. The binaries could be of two major types:

(i) detached, separated WDWD binaries whose evolution is driven by radiation reaction. The gravitational wave (GW) carries information about the mass of the binary and the distance. 
(ii) interacting binaries, these are close systems with a significant tidal interaction and/or with the Roche lobe overflow. In these systems, the gravitational radiation reaction competes against mass transfer, and the orbital period can either increase or decrease [1].

The GW signals from the Galactic binaries are so numerous below $3 \mathrm{mHz}$ that they are not individually resolvable and form a cyclo-stationary background which dominates over the instrumental noise above $0.1 \mathrm{mHz}$. It was demonstrated [2] that one can detect and remove tens of thousands of those signals. The resolved systems will provide the map of the compact binaries in the Galaxy and will allow us to constrain the evolutionary pathways of those systems.

Currently, two data analysis methods can be successfully demonstrated: the Bayesian method which utilizes the Markov Chain Monte Carlo with the Metropolis-Hastings rejection/acceptance rule [2] and the method which uses a bank of templates [3]. All methods use matched filtering in the form of the $\mathcal{F}$-statistic. Here, we present the method which also utilizes the template bank, but implementation is very different from [3]. The authors in [3] use the semi-coherent method adopted from LIGO-VIRGO data analysis and detect multiple signals in one go through the template bank (everything above a certain threshold), and then deal with secondary maxima at the post-analysis stage.

In the present method, we use two data streams with uncorrelated noise and perform fully coherent matched filtering on the network. We construct the template bank so that the nodes of the grid coincide with the Fourier frequencies, which allows us to compute the $\mathcal{F}$-statistic using the fast Fourier transform (FFT) algorithm. We use the FFT implementation provided by the FFTW project (http://www.fftw.org/). After filtering the data through the template bank, we refine the search using the Nelder-Mead procedure seeded at the grid point which gave us the maximum $\mathcal{F}$-statistic. Then we remove the signal from the data and repeat the procedure.

This paper is organized as follows. In section 2, we briefly present the linear phase approximation model that enables the fast evaluation of the detection statistic for GW signals from nearly monochromatic sources by using the FFT algorithm. In section 3, we define the covering problem with constraint and present a construction of nearly optimal lattices in any number of dimensions. In section 4, we present our search strategy to extract white dwarfs binaries from the MLDC3 data set. In the appendix, we briefly summarize some basic definitions from the theory of lattices.

\section{The optimal statistic}

The response of the space-based detector LISA to gravitational wave form Galactic binaries can be written $[4,5]$ as a linear combination of four functions $h^{(k)}$ depending on time. Each function $h^{(k)}$ depends on parameters $\xi^{\mu}$ called intrinsic parameters and the constant coefficients $a^{(k)}$ called extrinsic parameters:

$$
h\left(t ; a^{(k)}, \xi^{\mu}\right)=\sum_{k=1}^{4} a^{(k)} h^{(k)}\left(t ; \xi^{\mu}\right) .
$$

In the maximum-likelihood search method, the estimators $\left(\hat{a}^{(k)}, \hat{\xi}^{\mu}\right)$ are found by maximizing the $\log$ likelihood function with respect to parameters $\left(a^{(k)}, \xi^{\mu}\right)$. By solving explicitly the maximum likelihood equation for the extrinsic parameters, one can define the so-called $\mathcal{F}$ statistic and reduce the search to the intrinsic parameters space. For a signal from whitedwarf binaries buried in a stationary Gaussian noise and for intrinsic parameters defined by $\left(\xi^{1}, \xi^{2}, \xi^{3}, \xi^{4}\right)=(\omega, \dot{\omega}, \beta, \lambda)$, where $\omega$ is the angular frequency of the gravitational wave, 
$\dot{\omega}$ is the time derivative of $\omega, \beta$ and $\lambda$ are the latitude and the longitude of the source, the detection statistic $\mathcal{F}$ is given by

$$
\mathcal{F} \cong \frac{2}{S_{o} T_{o}}\left(V\left|\mathcal{N}_{u}\right|^{2}+U\left|\mathcal{N}_{v}\right|^{2}-2 \Re\left(W \mathcal{N}_{u} \mathcal{N}_{v}^{*}\right)\right)
$$

where $T_{o}$ is the observation time, $S_{o}$ is one-sided spectral density around the frequency of the signal, $V, U, W$ are normalization functions and $\mathcal{N}_{u}, \mathcal{N}_{v}$ are integrals given by

$$
\begin{aligned}
& \mathcal{N}_{u}=\int_{0}^{T_{o}} x(t) u(t ; \omega, \beta, \lambda) \exp \left[\mathrm{i} \phi_{\text {mod }}(t ; \omega, \dot{\omega}, \beta, \lambda)\right] \exp \mathrm{i} \omega t \mathrm{~d} t, \\
& \mathcal{N}_{v}=\int_{0}^{T_{o}} x(t) v(t ; \omega, \beta, \lambda) \exp \left[\mathrm{i} \phi_{\bmod }(t ; \omega, \dot{\omega}, \beta, \lambda)\right] \exp \mathrm{i} \omega t \mathrm{~d} t
\end{aligned}
$$

where $x(t)$ are the noisy data, $u, v$ are amplitude modulation functions and $\phi_{\text {mod }}$ is the phase modulation function. Since the modulation functions $u, v$ and $\phi_{\bmod }$ depend on frequency $\omega$, we cannot apply the FFT algorithm directly to calculate the $\mathcal{F}$-statistic. In order to do that, we first analyze the data in the narrow bands over which the slowly varying modulation functions $u$ and $v$ are evaluated at the mid frequency of the band and, second, we introduce a linear parametrization of the phase

$$
\phi_{\text {mod }}=\frac{1}{2} \dot{\omega} t^{2}+A \cos \Omega t+B \sin \Omega t,
$$

where frequency is absorbed into the new parameters $A$ and $B$,

$$
\begin{aligned}
& A=\omega R \cos \beta \cos \left(\lambda-\eta_{0}\right) \\
& B=\omega R \cos \beta \sin \left(\lambda-\eta_{0}\right),
\end{aligned}
$$

where $\eta_{0}$ is the initial position of the LISA constellation on the orbit around the sun, $R=1$ AU and $\Omega=2 \pi / \mathrm{yr}$.

In order to construct a grid in the intrinsic parameter space over which we calculate the $\mathcal{F}$-statistic, we introduce the following approximation to the signal that we call the linear model:

$$
h(t)=A_{0} \cos \left(\omega t+\frac{1}{2} \dot{\omega} t^{2}+A \cos \Omega t+B \sin \Omega t+\phi_{0}\right),
$$

where $A_{0}$ is a constant amplitude and $\phi_{0}$ is a constant phase. The phase modulation of the linear model is exactly the same as that of the exact model (equation (2)) whereas the amplitude is constant. This is a reasonable approximation because the amplitude modulation function varies very slowly, they are periodic functions of one year. We first calculate the reduced Fisher matrix ([4] equation (45)) $\tilde{\Gamma}$ for the above signal. This is the normalized Fisher matrix projected on the space of the intrinsic parameters $(\omega, \dot{\omega}, A, B)$. When the observation time $T_{o}$ is an integer $n$ of years, the coefficients of $\tilde{\Gamma}$ are given by (in dimensionless units with $T_{o}=1$ )

$$
\tilde{\Gamma}_{i j}=\left(\begin{array}{cccc}
1 / 12 & 1 / 24 & 0 & -\frac{1}{2 \pi n} \\
1 / 24 & 1 / 45 & \frac{1}{4 \pi^{2} n^{2}} & -\frac{1}{4 \pi n} \\
0 & \frac{1}{4 \pi^{2} n^{2}} & 1 / 2 & 0 \\
-\frac{1}{2 \pi n} & -\frac{1}{4 \pi n} & 0 & 1 / 2
\end{array}\right) .
$$

We see that the coefficients of $\tilde{\Gamma}$ are constant, independent of the values of the parameters. Next, we assume $\tilde{\Gamma}$ to be a metric in the parameter space and use it to distribute points in the grid in such a way that the distance defined by $\tilde{\Gamma}$ from any point of the parameter space to the nearest node of the grid is not larger than some fixed value $r$. The relation between the 
distance $r$, the reduced Fisher matrix and the loss of the signal-to-noise ratio when parameters of the signal differ from the parameters of the template by $\Delta \vec{\theta}(\Delta \vec{\theta}=(\Delta \omega, \Delta \dot{\omega}, \Delta A, \Delta B))$ is given by $([4,7])$

$$
\tilde{\Gamma}(\Delta \vec{\theta}, \Delta \vec{\theta})=r^{2}=\frac{\rho^{2}(0)-\rho^{2}(\Delta \vec{\theta})}{\rho^{2}(0)}+\varnothing\left(|\Delta \vec{\theta}|^{3}\right),
$$

where $\rho^{2}(\Delta \vec{\theta})$ is the signal-to-noise ratio squared when parameters of the signal differ from the parameters of the template by $\Delta \vec{\theta}$.

\section{Covering problem with constraints}

In our method, the detection of weak, quasi-monochromatic GW signals relies on an efficient placement of the templates in the bank. It should minimize the number of templates for a given allowed loss in the signal-to-noise ratio due to the offset between true signal parameters and the template parameters. The problem of constructing a grid in a $d$-dimensional parameter space is equivalent to the problem of covering $d$-dimensional space with equal overlapping spheres of a given radius. The optimal covering would have minimal possible thickness (see [6] and appendix). When the metric is flat, as for the case of the linear model (equation (5)), centers of the spheres lie on a $d$-dimensional lattice. In this case, one can take advantage of the theory of lattice coverings. For example, the thinnest lattice coverings are known in dimensions up to 5 .

In our search scheme, the calculation of the $\mathcal{F}$-statistic involves two Fourier transforms that can be computed efficiently using the fast Fourier transform algorithm. For this reason, we want the nodes of the grid to coincide with Fourier frequencies: $\Delta \omega, 2 \Delta \omega, 3 \Delta \omega, \ldots$ for some fixed frequency resolution $\Delta \omega$. This imposes a condition that one of the lattice basis vectors has a fixed length

$$
|\vec{l}|=\sqrt{\tilde{\Gamma}[(\Delta \omega, 0, \ldots, 0),(\Delta \omega, 0, \ldots, 0)]}
$$

and forbids an immediate use of the general results of the theory of lattice coverings. Instead one can formulate the covering problem with constraint: to find the thinnest lattice covering of the $d$-dimensional space with spheres of radius $r$ and one of the basis vectors of the lattice having fixed length $|\vec{l}|$. As far as we know, the general solution to the problem is not known. We present construction of a lattice that satisfies the constraint with a good accuracy.

Let a vector $\vec{v}_{0}$ define the frequency resolution. We search for a lattice $\Lambda\left(w^{\prime}\right)$ of covering radius $R\left(\Lambda\left(w^{\prime}\right)\right)=r$ with lattice basis $w^{\prime}$ satisfying the constraints that can be expressed as $w^{\prime}=\left\{\vec{v}_{0}, \vec{w}_{1}^{\prime}, \ldots, \vec{w}_{d-1}^{\prime}\right\}$. We find an optimal constrained lattice starting with a thinnest unconstrained lattice in $d$-dimensions. The idea is to shrink the optimal lattice as little as possible such that one of the basis vectors of the resulting lattice coincides with the constraint vector $\vec{v}_{0}$.

We note first that for a given lattice $\Lambda$ there always exists the shortest lattice vector $\vec{l}$ satisfying $\left|\vec{v}_{0}\right| \leqslant \vec{l}$ that we denote by $\vec{l}(\Lambda)$. We define the following algorithm A:

$\frac{\text { AlgorithmA }}{\text { Input: Lattice } \Lambda ; \text { vector } \vec{v}_{0}}$
Output: Lattice $\Lambda^{\prime} ; \vec{l}\left(\Lambda^{\prime}\right)=\vec{v}_{0}$.
A1. Find $\vec{l}(\Lambda)$.
A2. Contract $\Lambda$ along $\vec{l}(\Lambda)$ to obtain $\Lambda_{c}$ with $\left|\vec{l}\left(\Lambda_{c}\right)\right|=\left|\vec{v}_{0}\right|$.
A3. Rotate $\Lambda_{c}$ to obtain a lattice $\Lambda_{r c}$ with $\vec{l}\left(\Lambda_{r c}\right)=\vec{v}_{0}$.
A4. Return $\Lambda^{\prime}=\Lambda_{r c}$.




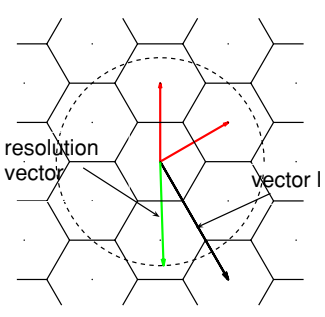

(a)

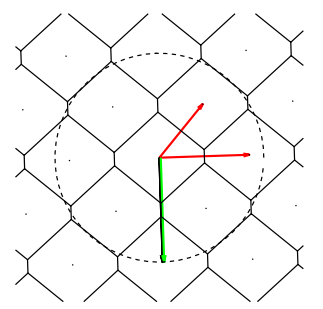

(c)

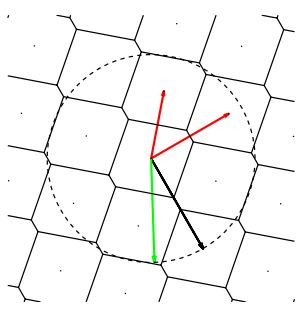

(b)

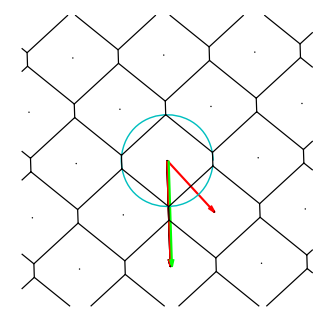

$(d)$

Figure 1. Illustration of the algorithm $\mathbf{A}$ in two dimensions. (a) Initial optimal lattice, (b) contraction of the lattice along vector $\vec{l},(c)$ rotation of the lattice and $(d)$ final suboptimal lattice with depicted covering radius.

The contraction of the initial lattice $\Lambda$ is 'minimal' in the sense that the thickness of the resulting lattice $\Lambda^{\prime}$ satisfies $\Theta(\Lambda) \leqslant \Theta\left(\Lambda^{\prime}\right) \leqslant \frac{|\vec{l}(\Lambda)|}{\left|\vec{v}_{0}\right|} \Theta(\Lambda)$.

The optimal constrained lattice is obtained by application of the algorithm $\mathbf{A}$ to an optimal (unconstrained) lattice $\Lambda$ with covering radius $r_{i}$ such that $R\left(\Lambda^{\prime}\right) \approx r$.

In dimensions $d=2,3,4,5$ as the initial lattices one takes the so-called Voronoi's principal lattice of the first type, $A_{d}^{*}[6]$ but the procedure can be generalized to any number of dimensions by taking as the input the best known lattice covering in a given dimension [6].

Figure 1 illustrates the procedure for the two-dimensional $A_{2}^{*}$ lattice. It is seen there that contraction of the lattice can change the initial Voronoi cell and the covering radius. However when the vector $\vec{l}(\Lambda)$ initially lies on the 'constraint surface' depicted by the dashed circle in figure 1 , the procedure acts trivially (no contraction only rotation) leaving the final lattice optimal. One often encounters trivial procedures when the vector $\vec{l}(\Lambda)$ is large as compared to the Voronoi cell, and moreover in this case contractions are small. On the other hand, the last trivial procedure occurs when the resolution vector and the shortest lattice vector have equal lengths, that is, when $R\left(A_{3}^{*}\right)^{2}=5 / 36 \pi^{2} \approx 1.3708$ and $R\left(A_{4}^{*}\right)^{2}=\pi^{2} / 6 \approx 1.6499$. For larger values, thickness of final lattices grows nearly linearly with the covering radius.

Some of these features are seen in figure 3 which shows results of application of the construction to the model (5) in 3 and 4 dimensions for different covering radii. The resolution vector is $\vec{v}_{0}=(2 \pi, 0, \ldots)$ and the observation time is 2 years. We do not continue above $r^{2}=1$ because of limited validity of the simple interpretation of the covering radius in terms of loss of the signal-to-noise ratio (see equation (7)). As for a fixed volume of the 


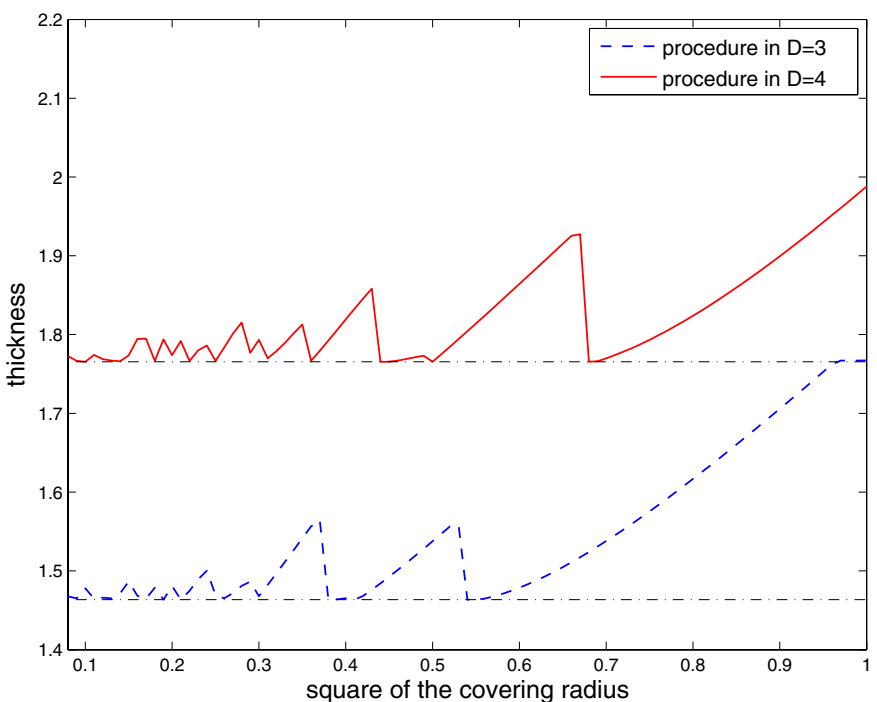

Figure 2. Thickness of the lattices in 3 and 4 dimensions compared to the optimal thickness of $A_{3}^{*} \approx 1.4635$ and $A_{4}^{*} \approx 1.7655$ as a function of the covering radius $r$. Relation between the covering radius and the maximum signal-to-noise ratio loss is given by equation (7).

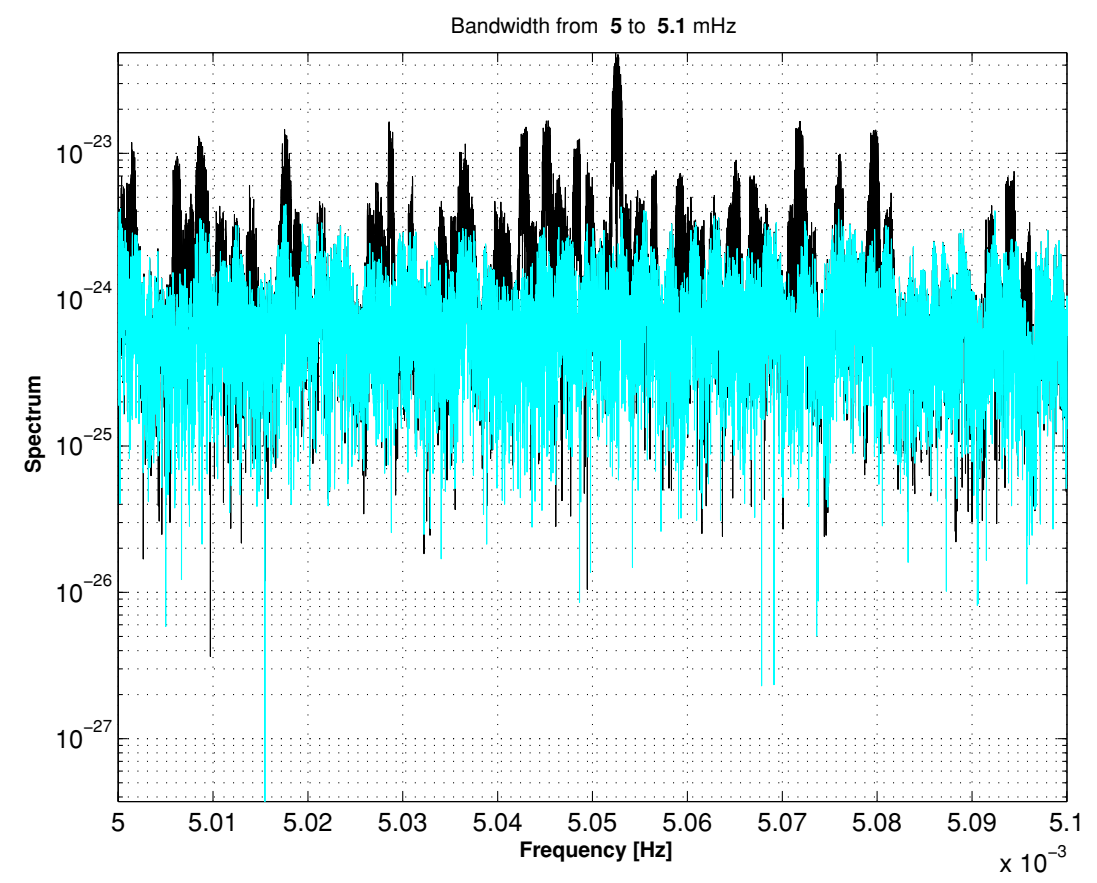

Figure 3. Estimation of the signals from white dwarf binaries in the training challenge 3.1 data set for the band from $5 \mathrm{mHz}$ to $5.1 \mathrm{mHz}$. Black color denotes the original data, and the light blue color is the data after signals removed. 


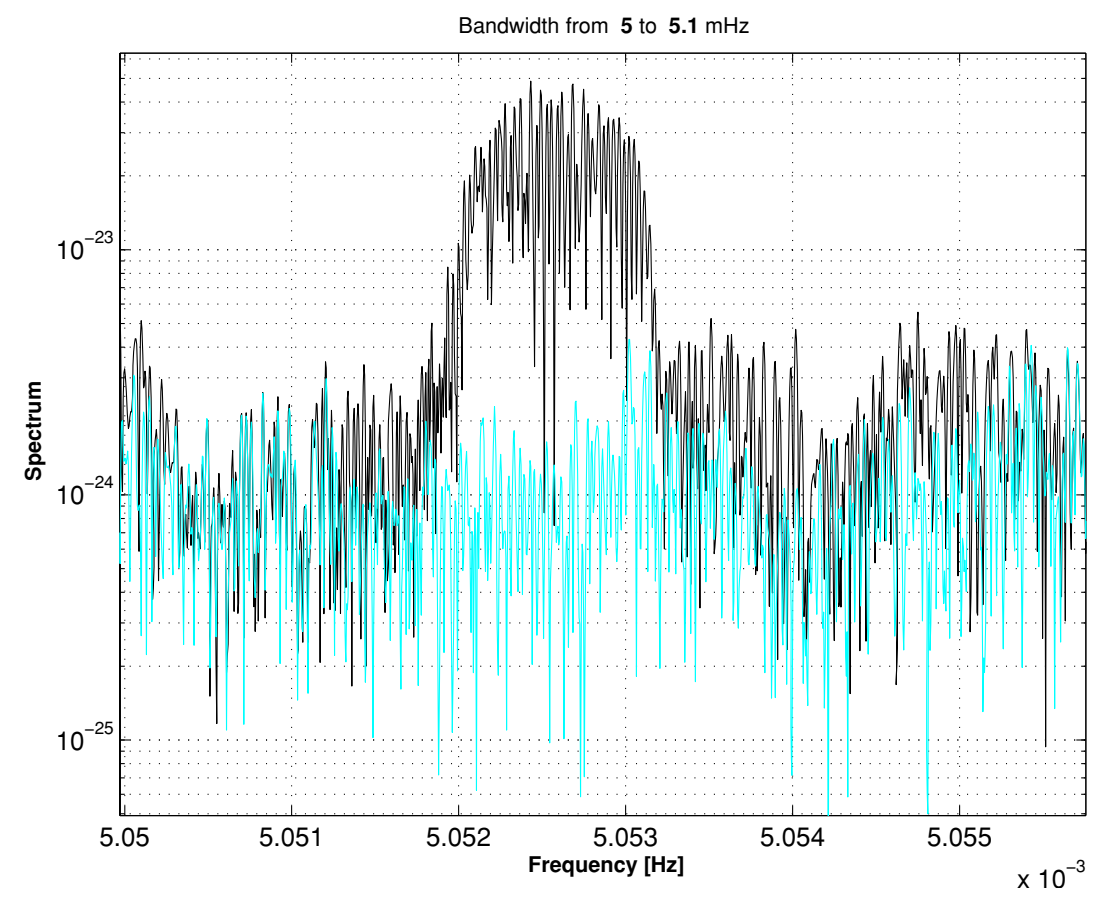

Figure 4. The zoom of figure 3 around the strongest signal identified.

parameter space, the number of points needed to cover the space with balls of a given radius (i.e., for allowed loss of the signal-to-noise ratio) is proportional to the thickness, the diagram demonstrates that the excess of points due to constraints is minor for the wide range of radii which makes the search strategy based on FFT/FFTW effective.

\section{Search strategy}

We have the following strategy to extract GW signals from WDWD binaries in the mock LISA data. We search the band from $0.1 \mathrm{mHz}$ to $25 \mathrm{mHz}$. We divide the data into bands of $0.1 \mathrm{mHz}$ each. Above $3 \mathrm{mHz}$, we include the frequency derivative in our search. In each band, we search for the signals using the $\mathcal{F}$-statistic and the following iterative procedure. We find the strongest signal, estimate its parameters, reconstruct the signal in the time domain and remove it from the data. We then search for the next strongest signal and so on until the signal-to-noise ratio (SNR) of the detected signal estimated as [4]

$$
\mathrm{SNR}=\sqrt{2(\mathcal{F}-2)}
$$

falls below a certain threshold. Estimation of parameters on the training data set led us to the following choice for the thresholds: for frequencies below $3 \mathrm{mHz} \mathrm{SNR}=10$ and for frequencies above $3 \mathrm{mHz}$ SNR $=7.5$. The choice of threshold is dictated by the computing resources available to do the analysis. To do the search, we use a constrained grid described in the previous section. For each signal, the coarse search over the grid is followed by a fine search using the Nelder-Mead procedure with the initial values provided by the template with the largest $\mathcal{F}$-statistic. In our analysis, we always search for individual signals without taking into account interference between the signals. In figures 3 and 4, we have presented 
application of the above strategy to the training challenge 3.1 data set in the bandwidth from $5 \mathrm{mHz}$ to $5.1 \mathrm{mHz}$.

In this bandwidth, we have identified 75 signals altogether out of 76 above the threshold of 7.5 present in the data. The total number of signals present in the bandwidth was 190 . The accuracy of estimation of most of the signals is one sigma, where the sigma is calculated from the Fisher matrix. For several signals, the error was very large indicating that either noise mimicked the signal or the residuals of removed signals remained significant. This could happen as a result of interference of the signals.

\section{Summary}

We have presented an efficient algorithm to search for almost monochromatic GW signals in the simulated LISA data. We construct a nearly optimal grid with constraint which allows us to use FFT in computing $\mathcal{F}$-statistic. We filter the data through the template bank. The template with the highest $\mathcal{F}$-statistic is used as a seed for the next step where we further refine the parameters. Then the strongest signal is removed from the data and the search procedure is repeated until the remaining signals have SNR below a preselected threshold. We have tested the algorithm on the training data set.

\section{Appendix A.}

In general for any discrete set of points $\mathcal{S}=\left\{\vec{s}_{1}, \vec{s}_{2}, \ldots\right\}$ in $\mathbf{R}^{n}$ the covering radius $R$ of $\mathcal{S}$ is defined as the least upper bound for any point of $\mathbf{R}^{n}$ to the closest point $\vec{s}_{i}$ :

$$
R(\mathcal{S})=\sup _{\vec{x} \in \mathbf{R}^{n}} \inf _{\vec{s} \in \mathcal{S}}|\vec{x}-\vec{s}| .
$$

Then spheres of equal radius $r$ centered at the points $\vec{s}_{i}$ will cover $\mathbf{R}^{n}$ only if $r \geqslant R$.

A lattice $\Lambda$ is a discrete subset of $\mathbf{R}^{n}$. Any lattice has a basis $\left\{\vec{l}_{1}, \ldots, \vec{l}_{n}\right\}$ of linearly independent vectors such that the lattice is the set of all linear combinations of $\vec{l}_{i}$ 's with integer coefficients:

$$
\Lambda=\left\{\sum_{i=1}^{n} c_{i} \vec{l}_{i}: c_{i} \in \mathbf{Z}, \quad i=1,2, \ldots, n\right\} .
$$

A lattice basis is not unique, in dimensions $d>1$ there are infinitely many of them, but all the bases have the same number of elements called the dimension of the lattice.

The parallelotope consisting of points $c_{1} \vec{l}_{1}+\cdots+c_{n} \vec{l}_{1}$ with $0 \leqslant c_{i}<1$ is a fundamental parallelotope and is an example of an elementary cell, that is, the building block containing one lattice point which tiles the whole $\mathbf{R}^{n}$ by translations of lattice vectors. There are infinitely many elementary cells but the volume of each elementary cell is unique for a given lattice $\Lambda$.

The Voronoi cell around any point $\vec{v}$ of $\Lambda$ is the set of vectors $\vec{x}$ of $\mathbf{R}^{n}$ which are closer to $\vec{v}$ than to any other lattice vector:

$$
V(\vec{v})=\{\vec{x}:|\vec{x}-\vec{w}| \geqslant|\vec{x}-\vec{v}| \text { for all } \vec{w} \in \Lambda\} .
$$

All Voronoi cells of a given lattice are congruent convex polytopes and are other examples of elementary cells sometimes referred to as Wigner-Seitz cells or Brillouin zones.

For the lattice $\Lambda$ having Voronoi cells congruent to polytope $V(\vec{v})$, where $\vec{v}$ is any of the lattice points, the covering radius is the circumradius $R(\Lambda)$ of $V(\vec{v})$, i.e., the largest distance between $\vec{v}$ and the vertices of $V(\vec{v})$. 
The thickness of the lattice covering is given by

$$
\Theta(\Lambda)=\frac{\text { volume of } d \text {-dimensional sphere of radius } R(\Lambda)}{\text { volume of the elementary cell of } \Lambda} .
$$

\section{References}

[1] Nelemans G, Yungelson L R and Portegies Zwart S F 2001 The gravitational wave signal from the Galactic disk population of binaries containing two compact objects Astron. Astrophys. 375890898

Nelemans G, Yungelson L R and Portegies Zwart S F 2004 Short-period AM CVn systems as optical, x-ray and gravitational-wave sources Mon. Not. R. Astron. Soc. 349181192

[2] Crowder J and Cornish N J 2007 Extracting galactic binary signals from the first round of Mock LISA Data Challenges Class. Quantum Grav. 24 S575-86

Babak S et al 2008 Report on the second Mock LISA Data Challenge Class. Quantum Grav. 25114037

[3] Prix R and Whelan J T 2007 F-statistic search for white-dwarf binaries in the first Mock LISA Data Challenge Class. Quantum Grav. 24 S565-74

Whelan J T, Prix R and Khurana D 2008 Improved search for galactic white dwarf binaries in Mock LISA Data Challenge 1B using an F-statistic template bank Class. Quantum Grav. 25184029

[4] Jaranowski P and Królak A 2005 Gravitational-wave data analysis. Formalism and simple applications: the Gaussian case Living Rev. Rel. 83 (cited on 6 June 2008): http://www.livingreviews.org/lrr-2005-3

[5] Królak A, Tinto M and Vallisneri M 2004 Optimal filtering of the LISA data Phys. Rev. D 70022003

[6] Conway J H and Sloane N J A 1993 Sphere Packings, Lattices and Groups (New York: Springer)

[7] Prix R 2007 The search for continuous gravitational waves: metric of the multi-detector $\mathcal{F}$-statistic Phys. Rev. D 75023004 